\title{
Performance and Security Evaluation of AES S-Box-based Glitch PUFs on FPGAs
}

\author{
Dai Yamamoto ${ }^{1,2}$, Gabriel Hospodar ${ }^{1}$, Roel Maes $^{1}$, Ingrid Verbauwhede ${ }^{1}$ \\ 1 KU Leuven ESAT/SCD-COSIC and IBBT \\ Kasteelpark Arenberg 10, B-3001 Leuven-Heverlee, Belgium \\ firstname.lastname@esat.kuleuven.be \\ ${ }^{2}$ FUJITSU LABORATORIES LTD. \\ 4-1-1, Kamikodanaka, Nakahara-ku, Kawasaki, 211-8588, Japan \\ ydai@labs.fujitsu.com
}

\begin{abstract}
Physical(ly) Unclonable Functions (PUFs) are expected to represent a solution for secure ID generation, authentication, and other important security applications. Researchers have developed several kinds of PUFs and self-evaluated them to demonstrate their advantages. However, both performance and security aspects of some proposals have not been thoroughly and independently evaluated. Third-party evaluation is important to discuss whether a proposal performs according to what the developers claim, regardless of any accidental bias. In this paper, we focus on Glitch PUFs (GPUFs) that use an AES S-Box implementation as a glitch generator, as proposed by Suzuki et al. [1]. They claim that this GPUF is one of the most practically feasible and secure delay-based PUFs. However, it has not been evaluated by other researchers yet. We evaluate GPUFs implemented on FPGAs and present three novel results. First, we clarify that the total number of challenge-response pairs of GPUFs is $2^{19}$, instead of $2^{11}$. Second, we show that a GPUF implementation has low robustness against voltage variation. Third, we point out that the GPUF has "weak" challenges leading to responses that can be more easily predictable than others by an adversary. Our results indicate that GPUFs that use the AES S-Box as the glitch generator present almost no PUF-behavior as both reliability and uniqueness are relatively low. In conclusion, our case study on FPGAs suggests that GPUFs should not use the AES S-Box as a glitch generator due to performance and security reasons.
\end{abstract}

Keywords: Glitch PUF, FPGA, Security, Performance, Key Generation, Authentication.

\section{Introduction}

Secure identification/authentication technology using integrated circuits (ICs) is very important for a secure information infrastructure. One is often concerned with finding solutions for anti-counterfeiting devices on medical supplies, prepaid-cards and public ID cards such as passports and driver's licenses. The 
IC card is a well-known solution for this kind of application. Counterfeiting is prevented by storing a secret key on the IC card and using a secure cryptographic protocol to make the key invisible to the outside. In theory, however, the possibility of counterfeiting still remains if the IC design is revealed and reproduced. Recently, interest has been focused on Physical(ly) Unclonable Functions (PUFs) as a solution to the aforementioned issue [2]. In a PUF realized in an IC (silicon PUF), the output value (response) to the input value (challenge) is unique for each individual IC. This uniqueness is provided by random process variations that occur in the manufacturing process of each IC [3] [4]. It is expected that PUFs will represent a breakthrough in technology for anti-counterfeiting devices through its use for ID generation, key generation and authentication protocols, making cloning impossible even when the design is revealed.

The silicon PUFs are basically classified into two categories [5]. One uses the characteristics of memory cells such as SRAM-PUFs [6] [7], Butterfly PUFs [8], Flip-flop PUFs [9], Mecca PUFs [10] and Latch PUFs [11] [12]. The other uses the characteristics of delay variations such as Ring Oscillator PUFs [13], Arbiter PUFs [14] and Glitch PUFs (GPUFs) [1]. This paper focuses on the latter. Ring Oscillator PUFs derive entropy from the difference in oscillator frequencies. Arbiter PUFs have an arbiter circuit that generates a response determined by the difference in the signal delay between two paths set by a challenge. However, a machine learning attack can predict responses of Arbiter PUFs by using a number of challenge-response pairs (CRPs), as it has been shown that the relationship between challenges and responses is linear [15]. The GPUF [1] was proposed to solve this problem of ease of prediction. A glitch is a pulse of short duration which may occur before the signal settles to a value. The GPUF generates a one-bit response by using the parity of the number of glitches obtained from an 8-bit AES S-Box implementation used as a glitch generator. Part of the challenges correspond to 8-bit inputs to the S-Box. Since the response to challenges behaves like a non-linear function, the developers claim that machine learning attacks are prevented.

Although PUF developers evaluate their proposals themselves, some of them may either accidentally exaggerate on good results or not mention undesirable ones. Hence it is quite important not only to propose and evaluate new PUFs, but also to get the proposals evaluated and analyzed by third-party researchers.

Our Contributions In this paper, we evaluate both performance and security aspects of the GPUF developed by Suzuki et al. [1] (i.e. "developers") implemented on FPGAs. The reason why we focus on this PUF is that it is one of the most feasible and secure delay-based PUFs because of the resistance against machine learning attacks. However, it has not been evaluated by other researchers yet. Our main contribution consists of three parts. First, we propose a general method to generate responses because the original paper is somewhat obscure about it. To the best of our knowledge, the developers used only $2^{8}$ challenges as input to the 8-bit AES S-Box glitch generator. Hence they relied on a total of $256 \times 8=2,048$ responses since the AES S-Box has 81 -bit outputs. We point 
out that glitches normally appear when an 8-bit input value of the S-Box is transitioned from one value to another. The glitches thus depend on the input values both before and after the transition. Consequently, a GPUF based on an 8-bit AES S-Box has $256 \times 256 \times 8=2^{19} \mathrm{CRPs}$. It means that the performance results presented by the developers are insufficient as they evaluated only a subset of all CRPs. Second, we evaluate the performance of GPUFs using all CRPs. We clarify that both reliability and uniqueness strongly depend on the Hamming distance between the AES S-Box input values before and after the transition. Therefore, GPUF designers have to carefully select the set of CRPs meeting their security requirements, which increases design costs. Additionally, if the supply voltages are changed within the rated voltage range of FPGAs $(1.14 \mathrm{~V} \sim 1.26 \mathrm{~V})$, GPUFs present low reliability - meaning that the intra-chip variation is greater than $30 \%$. This value exceeds the error correction range when using a Fuzzy Extractor with a reasonable size of redundant data. This indicates that GPUFs present almost no PUF-behavior. Third, we analyze the security of GPUFs. If the AES S-Box input value after the transition is chosen to be one out of 16 specific values, then the number of glitches is almost zero regardless of the input value before the transition. AES S-Box-based GPUFs have "weak" challenges (like a weak key for a block cipher) leading to responses that are more easily predictable than others by an attacker, which could compromise the whole security of a GPUF-based system.

Organization of the Paper The rest of the paper is organized as follows. Section 2 gives an outline of the original GPUF proposed by the developers, and our proposed method to generate responses using all CRPs. Section 3 evaluates the performance of the GPUF implemented on an FPGA platform. We evaluate both reliability and uniqueness in various voltages. Section 4 evaluates the security of the GPUF, and discusses weak challenges that should not be used. Finally, in Section 5 we summarize our work and comment on future directions.

\section{Glitch PUF}

\subsection{Original GPUF Proposal by Suzuki et al. [1] [16]}

Different GPUFs have been proposed until now. In 2008, Crouch et al. [17] [18] first proposed the concept of extracting a unique digital identification using glitches obtained from a 32-bit combinational multiplier. In 2010, Anderson [19] proposed a glitch-based PUF design specifically targeted for FPGAs. This GPUF generates a one-bit response based on the delay differences between two multiplexer (MUX) chains. Then, a new glitch-based PUF using one AES S-Box as a glitch generator was proposed in 2010 [1], and improved in 2012 [16] by Suzuki et al. In this paper, we focus only on the third GPUF proposal (and refer to it as only GPUF) because of its good performance, good security features such as resistance against machine learning attacks, and practical advantages as it can be implemented on ASIC and FPGA platforms, as claimed by the authors. 
Figure 1 presents this GPUF. It uses one 8-bit AES S-Box based on composite Galois field as a glitch generator. The challenge input to the GPUF has 11 bits and is composed of two parts. The first part of the challenge contains 8 bits inputted from the data registers to the AES S-Box. Each of the 8 output bits of the S-Box generates a different number of glitches due to the complicated nonlinearity of the AES S-Box implementation. The second part of the challenge contains 3 bits to select one out of the 8 AES S-Box output bits. A toggle flipflop (TFF) eventually outputs the GPUF response by evaluating the parity of the number of glitches that appear in the selected AES S-Box output bit. To the best of our knowledge, the developers have evaluated $2^{11}$ CRPs. The masking scheme is used to select stable challenges that output the same responses at normal operating condition (room temperature and standard supply voltage) most of the times. For each challenge, the developers evaluated its response 10 times. A challenge was considered stable if all 10 responses were equal. According to their strict methodology, challenges yielding at least one different response were discarded.

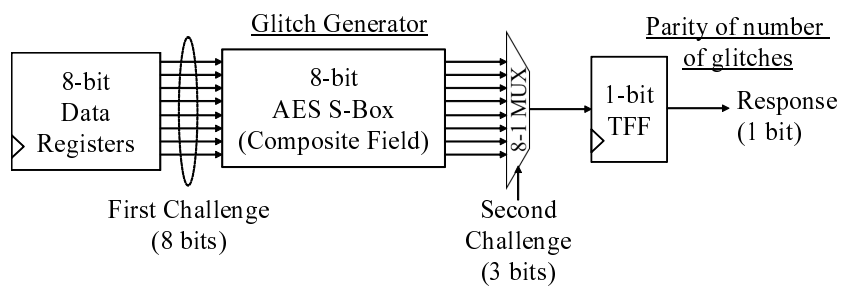

Fig. 1. Glitch PUF.

\subsection{Our Response Generation Method}

In this paper, glitches appear right after the first 8-bit part of the challenge is transitioned from one value (previous 8-bit challenge: $C_{\mathrm{p}}$ ) to another (current 8-bit challenge: $C_{\mathrm{C}}$ ). Figure 2 depicts a conceptual explanation of two cases. For example, for the same value of $C_{\mathrm{c}}$ (e.g. 31), the number of glitches are respectively 5 or 2 for $C_{\mathrm{p}}$ equal to 246 or 97 . Actually, the number of glitches strongly depends on both $C_{\mathrm{p}}$ and $C_{\mathrm{c}}$ according to our experiments (details in Sect. 3). Therefore, we claim that the first part of the GPUF challenge has not 8 , but 16 bits ( 8 bits from $C_{\mathrm{p}}$ and 8 bits from $C_{\mathrm{C}}$ ). The combination of all values of $C_{\mathrm{p}}$ and $C_{\mathrm{C}}$ leads to $256 \times 256=65,536 \mathrm{CRPs}$ per S-Box output bit. However, if both $C_{\mathrm{p}}$ and $C_{\mathrm{c}}$ are equal, then no glitch occurs since there is no bit transition, making the responses always equal to zero. Thus, the valid number of CRPs is reduced to $256 \times 255=65,280$. As the second part of the challenge has 3 bits, the AES S-Box-based GPUF has in fact a total of $65,280 \times 2^{3}=522,240$ CRPs. 


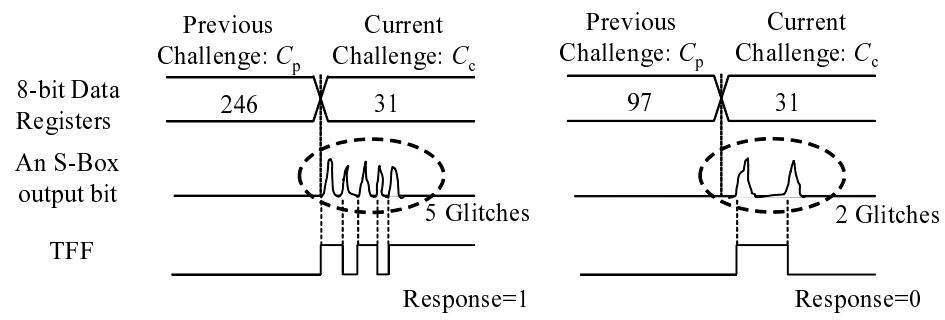

Fig. 2. Number of glitches with respect to $C_{\mathrm{p}}$ and $C_{\mathrm{c}}$.

\section{Performance Evaluation}

\subsection{Experimental Environment}

Figure 3 shows our experimental evaluation system, which uses a Spartan-3E starter kit board [20] with a Xilinx Spartan-3E FPGA (XC3S500E-4FG320C) and a custom-made expansion board with a Xilinx Spartan-6 FPGA (XC6SLX162CSG324C). The developers implemented both peripheral circuits such as the block RAM, RS232C module and GPUF circuit on the same FPGA chip. In contrast, we implement the peripheral circuits separately on a Spartan-3E (SP3E) FPGA, and the GPUF circuit on a Spartan-6 (SP6) FPGA. Such configuration enables us to change only the core voltage of the SP6 FPGA chip. The voltage change does not impact the peripheral circuits and does not cause data garbling, which enhances the confidence of our experimental results. An SP6 FPGA chip is put on a socket of the expansion board, being therefore easily replaceable by another chip. A programmable ROM (PROM) is implemented on the expansion board, allowing us to download our circuit design on the PROM through a JTAG port. The core voltage of an SP6 chip can be changed by $0.01 \mathrm{~V}$ using a stabilized power supply. The two boards are connected with user I/O interfaces through a connector. The clock signal is provided from the SP3E to the SP6 through a SMA cable and port in order to prevent signal degradation. A micro SD adapter and card are also connected to the SP3E board to store the responses from the GPUF. We evaluate 20 GPUFs implemented on 20 SP6 FPGA chips.

Figure 4 shows the details of our circuit designs realized on the SP3E and SP6 FPGA chips. The AES S-Box implementation based on composite Galois field techniques was obtained from the RTL code from [21]. A 50-MHz clock signal generated by an on-board oscillator is applied to a Digital Clock Manager (DCM) primitive yielding a 2.5-MHz clock signal that is applied to the GPUF. The data acquisition process is as follows. When the RS232C module from the SP3E chip receives a start command from a user PC, the module sends a start signal to the CTRL module. The module initializes the values of $C_{\mathrm{p}}$ and $C_{\mathrm{C}}$ to zero, and stores them into two registers dedicated for $C_{\mathrm{p}}\left(P_{1} \sim P_{8}\right)$ and $C_{\mathrm{C}}$ $\left(C_{1} \sim C_{8}\right)$ on the SP6, respectively. After that, registers storing the inputs to the 


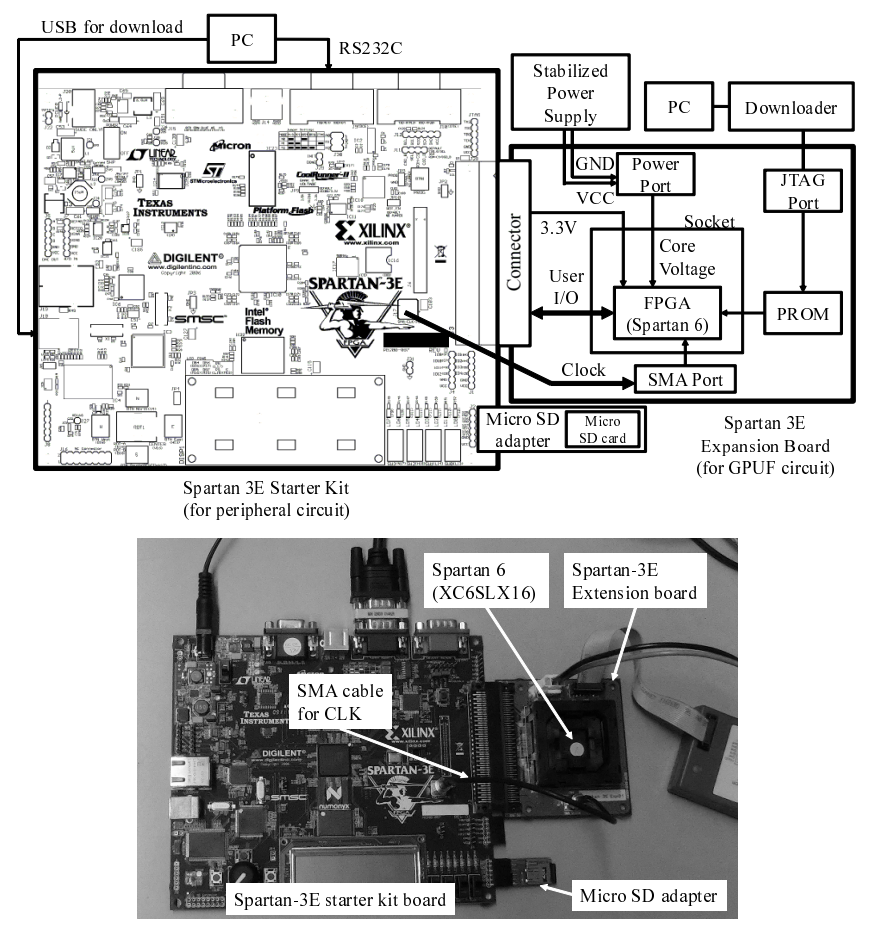

Fig. 3. Experimental evaluation system.

S-Box $\left(R_{1} \sim R_{8}\right)$ are transitioned from $C_{\mathrm{p}}$ to $C_{\mathrm{c}}$ in one cycle. We evaluate not the parity but the actual number of glitches output from the glitch generator. This does not influence the GPUF performance. The number of glitches is stored into eight 8-bit counters with TFFs $\left(T_{1,1} \sim T_{8,8}\right)$. Then, the total amount of 64 bits coming from eight 8-bit counters are sent to a block RAM on the SP3 bitsequentially. The values of the block RAM are sent to a SD write module, and written into a micro SD card. This process is repeated with the same $C_{\mathrm{p}}$ and $C_{\mathrm{C}}$ 100 times as in [1] [16]. Then both $C_{\mathrm{p}}$ and $C_{\mathrm{c}}$ are incremented by 1 from 0 to 255 and the process is repeated 100 times analogously. Note that the responses are meaningful when $C_{\mathrm{p}}$ is not equal to $C_{\mathrm{c}}$, as mentioned in Sect. 2.2.

In Sect. 3.2, we evaluate the following performance-related figure of merits [22] of GPUFs operating at 1.20V: reliability, uniqueness, uniformity and bit-aliasing. We choose $1.20 \mathrm{~V}$ as the standard voltage because the rated voltage range of the SP6 FPGA (XC6SLX16-2CSG324C) is $1.20 \pm 0.06 \mathrm{~V}(1.14 \mathrm{~V} \sim$ $1.26 \mathrm{~V})$. In the standard voltage of $1.20 \mathrm{~V}$ our GPUF implementations present performance results in accordance with the developers' ones. Later, in Sect. 3.3 we evaluate our GPUF implementations operating at the maximum allowed FPGA rated voltages of $1.14 \mathrm{~V}$ and $1.26 \mathrm{~V}$. 


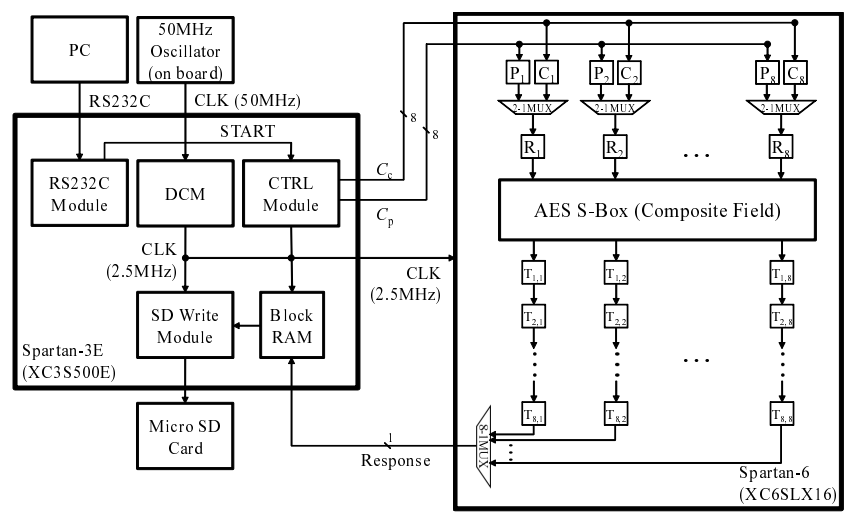

Fig. 4. Circuit design on FPGAs.

\subsection{Performance at the Standard Voltage of $1.20 \mathrm{~V}$}

The reliability and uniqueness results of our GPUF implementations are shown in Figs. 5 and 6, respectively. In order to evaluate the reliability, 101 responses are generated per SP6 FPGA chip (see Appendix). One response is used as the reference, and the remaining are used for analysis. The response space size is $65,280 \times 8$ bits. Figure 5 shows a histogram of normalized Hamming distances between the reference response and each repeated one (i.e. $100 \times 20$ (chips) $=$ 2,000 elements). The average error rate when masking is on is approximately $1.38 \%$ with a standard deviation (S.D.) of $0.11 \%$, which is much less than the $15 \%$ assumed in [23] for stable responses based on a Fuzzy Extractor with a reasonable size of redundant data. Hence our result shows that the GPUF yields highly reliable responses, in accordance with the developers' results. Next, in order to evaluate the uniqueness, a total of 20 responses using all 20 FPGAs (one response per FPGA) is generated. Figure 6 shows a histogram of normalized Hamming distances between every combination of two responses, i.e. ${ }_{20} \mathrm{C}_{2}=190$ combinations. This evaluation is a general way of showing the extent to which the responses of the chips are different. The difference in the responses of two arbitrary PUFs is approximately $39.8 \%$ with a S.D. of $1.1 \%$ when masking is on. GPUF yields responses with a lower level of uniqueness than the ideal difference of $50 \%$. This result also corresponds to the developers' one.

Next, we evaluate both the uniformity and bit-aliasing of GPUFs - a contribution that has not been addressed by the developers in [1] [16]. The uniformity evaluates how uniform the proportion of ' 0 's and ' 1 's is in the response bits of a PUF. For our GPUF implementations, the average uniformity is approximately $50.6 \%$ and $50.7 \%$ when masking is off and on, respectively. Since the ideal uniformity is $50 \%$, our GPUFs satisfy the requirement for uniformity. The bit-aliasing evaluates how different the proportion of ' 0 's and ' 1 's is in the 20 response bits extracted respectively from the 20 PUFs given the same challenge. 


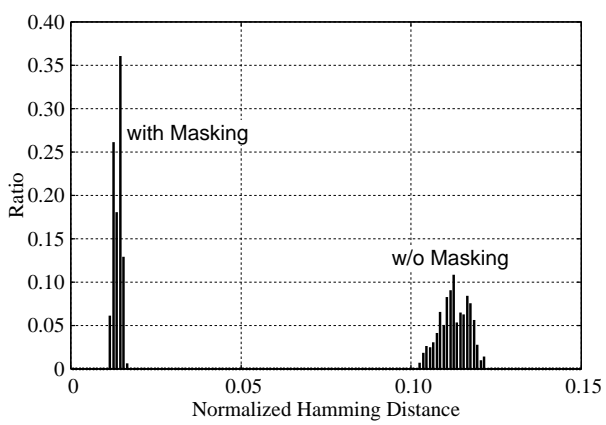

Fig. 5. Reliability.

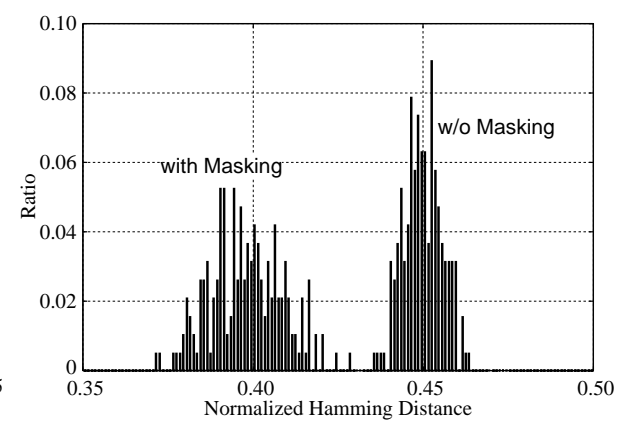

Fig. 6. Uniqueness.

The ideal bit-aliasing is also $50 \%$ with a S.D. of $0 \%$. Figures 7 (I) and (II) show histograms of the proportion of ' 1 's when masking is off and on, respectively. The bit-aliasing S.D. is approximately $4.7 \%$ larger when masking is used than when it is not used. This is because the masking scheme discards the responses whose proportion of ' 1 's is around $50 \%$. Hence Fig. (II) lacks the peak of the normal distribution. It turns out that there are many responses fixed to 0 or 1 in the GPUF implementations on the 20 chips. This means that GPUFs have many useless CRPs due to the predictability of the responses. Hence GPUF designers should not use all CRPs due to security reasons. This result is implied by the low uniqueness of GPUFs as shown in Fig. 6. The fact that the S.D. becomes larger when masking is being used is related to the lower uniqueness and entropy of responses, as previously mentioned by the developers.

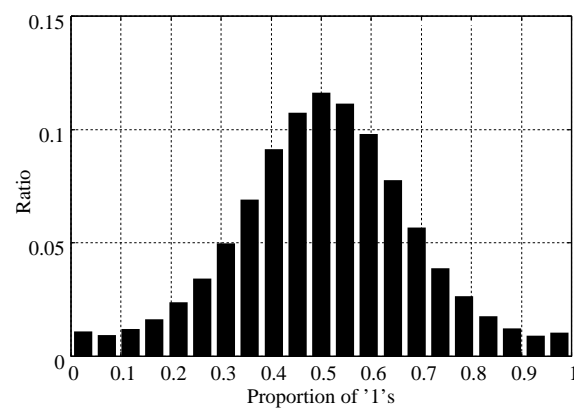

(I) Without Masking $($ Mean $=50.6 \%$, S.D. $=19.0 \%)$.

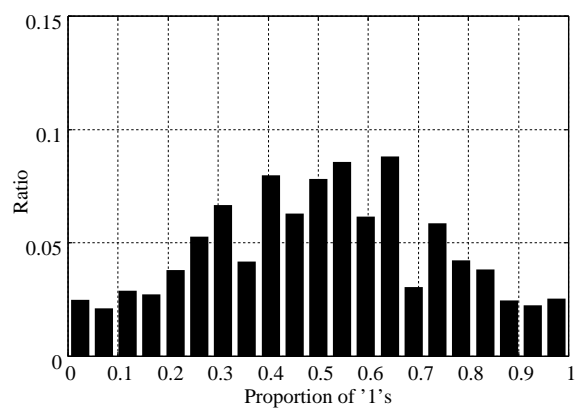

(II) With Masking

$($ Mean $=50.8 \%$, S.D. $=23.7 \%)$.

Fig. 7. Bit-aliasing. 


\subsection{Performance at Non-Standard Voltages $(1.14 \mathrm{~V}$ and $1.26 \mathrm{~V})$}

In this section, we evaluate the robustness of the GPUF against voltage variation - the reliability of GPUFs when their supply voltage is changed to $1.14 \mathrm{~V}$ and $1.26 \mathrm{~V}$. Figure 8 (I) shows the response error rates (see Appendix) of our GPUF implementations in comparison to the developers' ones. At $1.14 \mathrm{~V}$, our response error rate is approximately $35 \%$ when masking is on, differently from the developer's results $(\approx 10 \%)[16]$. A possible reason for the difference in the results could have been caused by our expansion board. However, the proper operation of our expansion board was verified by implementing Latch PUFs on the SP6 FPGAs and confirming that the response error rates are less than $15 \%$ even when changes in the supply voltage occur. Consequently, according to our evaluation, the robustness against voltage variation of GPUFs is much lower than the one provided by the developers. This is partly because they evaluated only $256 \times 8$ CRPs, while we consider all $256 \times 255 \times 8$ CRPs. In fact, if we choose only $256 \times 8 \mathrm{CRPs}$ satisfying the following two conditions: the Hamming distance between $C_{\mathrm{p}}$ and $C_{\mathrm{c}}$ being equal to $1\left(\mathrm{HD}\left(C_{\mathrm{p}}, C_{\mathrm{c}}\right)=1\right)$, and the different bit position being the least significant bit, then the robustness against voltage variation becomes remarkably better than the developers' results, as shown in Fig. 8 (II). In the following, we discuss the relationship between both reliability and uniqueness to the CRPs. The CRPs are divided into 8 groups based either on each value of $\operatorname{HD}\left(C_{\mathrm{p}}, C_{\mathrm{c}}\right)$ (excluding $\operatorname{HD}\left(C_{\mathrm{p}}, C_{\mathrm{c}}\right)=0$, as no glitches occur) or on which $\mathrm{S}$-Box bit is used to generate a response.

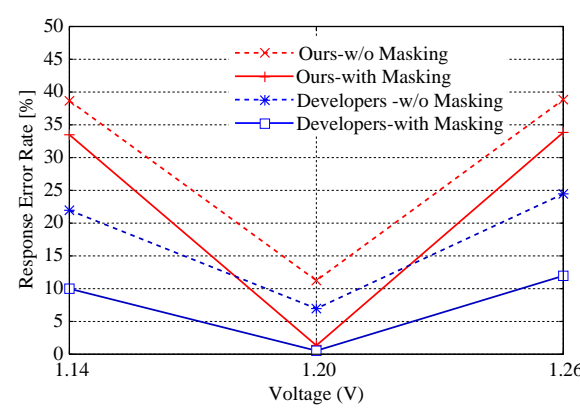

(I) Our vs. developers' results.

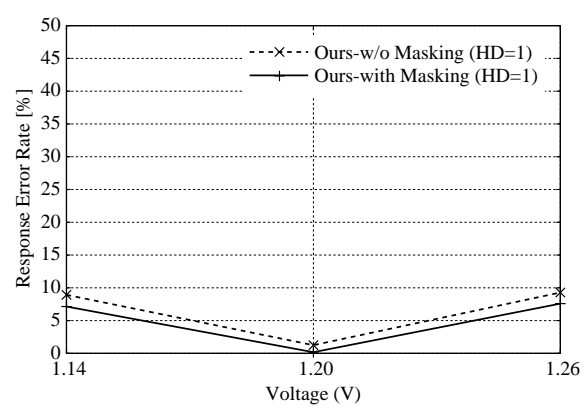

(II) Our results for $\operatorname{HD}\left(C_{\mathrm{p}}, C_{\mathrm{c}}\right)=1$.

Fig. 8. Response error rates against various voltages.

Figures 9 (I)-(III) and (IV) show the response error rates (reliability) and the uniqueness of CRPs extracted from each S-Box bit (S-Box $[0] \sim$ S-Box[7]), respectively. The reliability is evaluated at three voltages $(1.14 \mathrm{~V}, 1.20 \mathrm{~V}$ and $1.26 \mathrm{~V})$, while the uniqueness is evaluated only at the standard voltage of $1.20 \mathrm{~V}$. The results when masking is on and off are shown in the left and right histograms, respectively. At $1.14 \mathrm{~V}$ and $1.26 \mathrm{~V}$, the reliability ranges from 30 to $40 \%$ depending on the S-Box bit even when masking is on. At $1.20 \mathrm{~V}$, the uniqueness ranges from 
35 to $45 \%$ also depending on the S-Box bit when masking is on. The reliability and uniqueness distributions are thus close to each other, possibly overlapping. Therefore, our GPUF implementations show almost no PUF behavior as an authentication protocol free of errors cannot be implemented. As both reliability and uniqueness of GPUFs strongly depend on the S-Box bits used to generate responses, if GPUFs are used for key generation, suitable challenges should be carefully chosen based on security requirements.

Figures 10 (I) and (II) show the reliability and uniqueness of CRPs with respect to $\operatorname{HD}\left(C_{\mathrm{p}}, C_{\mathrm{C}}\right)=1, \ldots, 8$, respectively. Due to space constraints, we show: the average of 8 results (from S-Box[0] to S-Box[7]), the results of S-Box[2] (lowest reliability), and the results of S-Box[7] (highest reliability), as shown in Fig. 9 (I). The smaller $\operatorname{HD}\left(C_{\mathrm{p}}, C_{\mathrm{c}}\right)$ is, the higher the reliability is, and the lower the uniqueness is. This is because if $\operatorname{HD}\left(C_{\mathrm{p}}, C_{\mathrm{C}}\right)$ is small, the number of changed bits in the S-Box is also small. As a result, the transition from $C_{\mathrm{p}}$ to $C_{\mathrm{C}}$ has little influence on the generation of glitches. As GPUFs perform differently with regard to $\operatorname{HD}\left(C_{\mathrm{p}}, C_{\mathrm{C}}\right)$, the need for a designer to select appropriate CRPs meeting a system's requirement leads to an additional increase in the design cost. The reliability at $1.20 \mathrm{~V}$ can be dramatically enhanced by using the masking scheme proposed by the developers. However, the reliability cannot be enhanced effectively at $1.14 \mathrm{~V}$ and $1.26 \mathrm{~V}$ using the masking scheme. Consequently, there is no correlation between unstable CRPs at $1.20 \mathrm{~V}$ and at $1.14 \mathrm{~V}$ or $1.26 \mathrm{~V}$. GPUF designers should thus remove, i.e. mask, CRPs that are unstable not only at $1.20 \mathrm{~V}$ but also at $1.14 \mathrm{~V}$ and $1.26 \mathrm{~V}$. However, this is not realistic and practical. Such solution not only increases the manufacturing costs as well, but also reduces the number of CRPs, which causes loss of information entropy in the responses.

Finally, we evaluate the side effects of using the masking scheme: how many responses are unstable and therefore discarded. The three types of bar graphs in Fig. 11 show the number of stable responses in three cases: without masking (all responses), with masking at $1.20 \mathrm{~V}$ (stable responses at $1.20 \mathrm{~V}$ ) and with masking at three voltages (stable responses at $1.14 \mathrm{~V}, 1.20 \mathrm{~V}$ and $1.26 \mathrm{~V}$ ). In fact, the third case is not realistic and practical since the masking processes at all voltages have to be applied. We, however, show this case to evaluate the actual number of valid and stable CRPs in the GPUF. The line graphs in Fig. 11 show the ratio of stable responses in each group of $\operatorname{HD}\left(C_{\mathrm{p}}, C_{\mathrm{c}}\right)$. We show once more the average results for S-Box[2] and S-Box[7] due to space constraints, over the 20 $\mathrm{SP} 6$ FPGAs. The larger $\operatorname{HD}\left(C_{\mathrm{p}}, C_{\mathrm{C}}\right)$ is, the lower the ratio of stable responses is (i.e. the larger the number of discarded responses is). That is why the larger $\mathrm{HD}\left(C_{\mathrm{p}}, C_{\mathrm{C}}\right)$ is, the higher the response error rate is, as shown in Fig. 10 (I). Also, there are large gaps between the two lines in Figs. 11 (I)-(III). This means that the stable responses at $1.20 \mathrm{~V}$ are not always stable at $1.14 \mathrm{~V}$ and $1.26 \mathrm{~V}$. Hence the response error rate is high and the voltage resistance of the GPUF is quite low, as shown in Fig. 10 (I). By comparing Fig. 11 (II) and Fig. 11 (III), the lower the reliability is, the larger the number of discarded responses is. Out of a total of $65,280 \times 8$ responses, the ratios of stable responses at $1.20 \mathrm{~V}$ and at the three voltages are $61.7 \%$ and $30.1 \%$, respectively. Consequently, GPUFs 


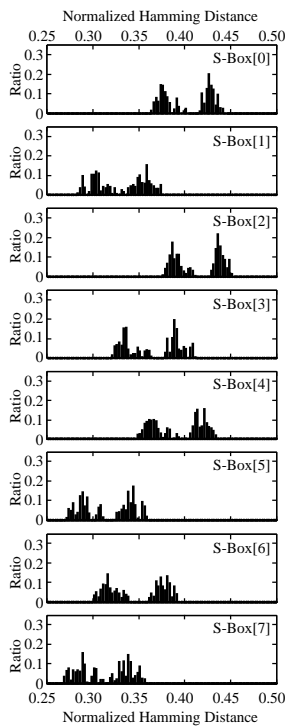

(I) Reliability at

$1.14 \mathrm{~V}$.

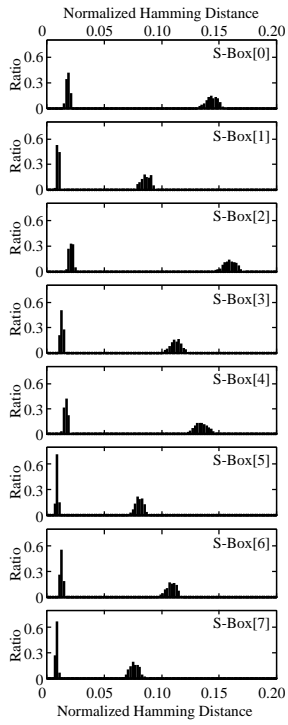

(II) Reliability at $1.20 \mathrm{~V}$.

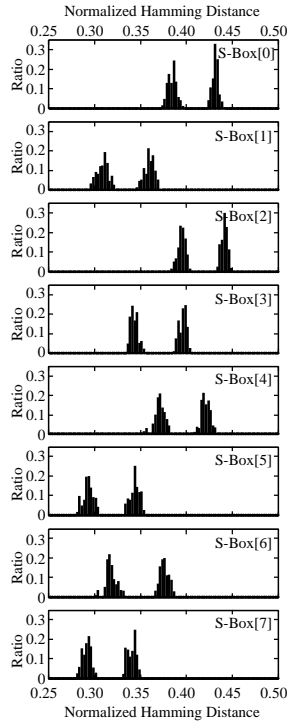

(III) Reliability at $1.26 \mathrm{~V}$.

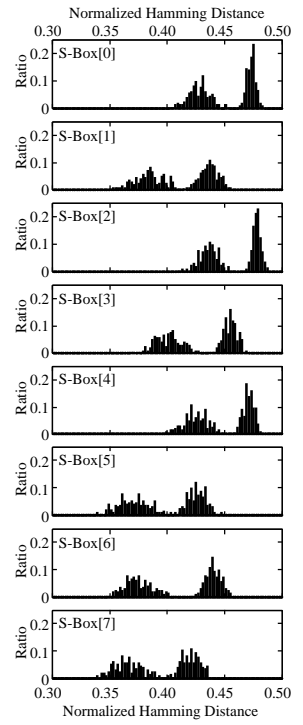

(IV) Uniqueness at $1.20 \mathrm{~V}$.

Fig. 9. Reliability and Uniqueness vs. S-Box output bits (left histograms: with masking, right: w/o masking).

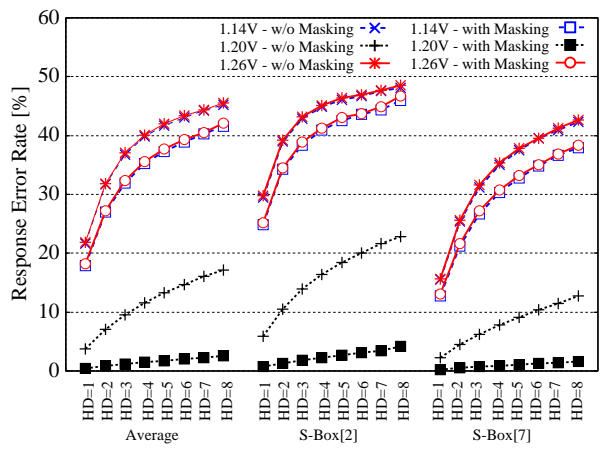

(I) Reliability.

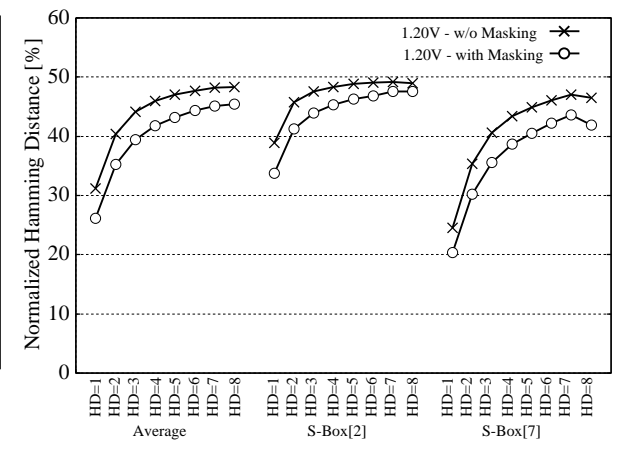

(II) Uniqueness.

Fig. 10. Reliability and Uniqueness vs. $\operatorname{HD}\left(C_{\mathrm{p}}, C_{\mathrm{c}}\right)$. 
have in fact a number of useless CRPs that should be removed by the masking scheme. This masking reduces the total number of CRPs or the total pattern of keys generated by multiple GPUFs. The low total number of CRPs or keys might facilitate an attacker to succeed in her modeling attack. In conclusion, our GPUFs implemented on FPGAs have a low robustness against voltage variation according to our evaluation results. In addition, both reliability and uniqueness strongly depend on the selected CRPs.

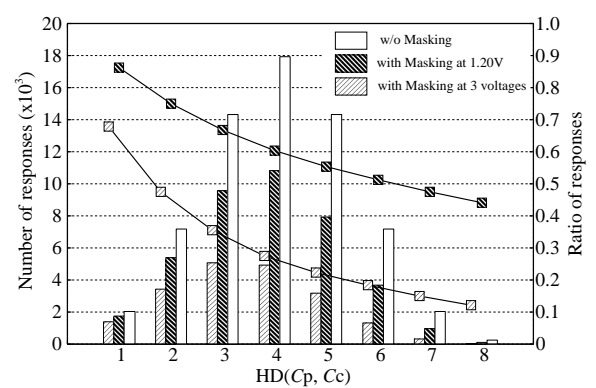

(I) Average (from S-Box[0] to S-Box[7])

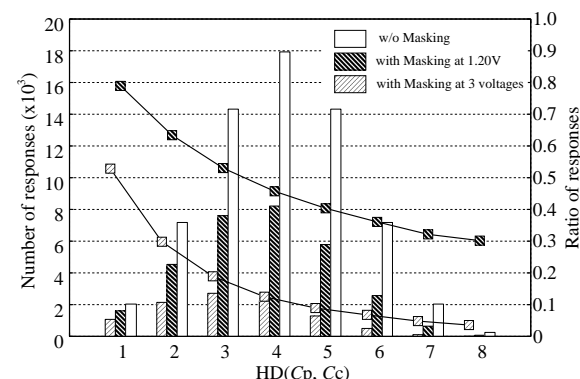

(II) S-Box[2] (lowest reliability)

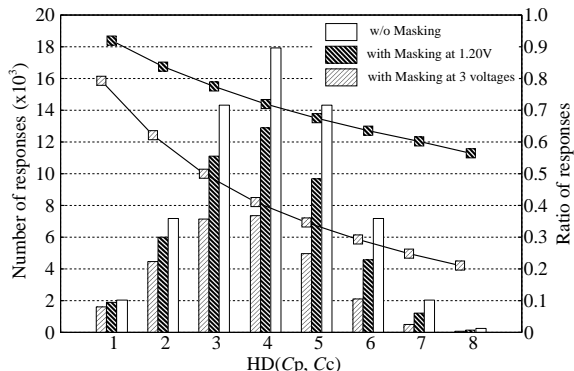

(III) S-Box[7] (highest reliability)

Fig. 11. Number and ratio of stable responses in three cases.

\section{Security Analysis}

In this section, we evaluate the security of AES S-Box-based GPUFs. Concretely, we clarify that the GPUF has "weak" challenges that are associated with more easily predictable responses. Figure 12 depicts the number of glitches generated from S-Box[6] on a single specific chip $(i=1)$. This figure represents a $256 \times$ 256 matrix, where the horizontal axis represents $C_{\mathrm{p}}$ and the vertical axis represents $C_{\mathrm{C}}$. Each element is colored from black to gray according to the number of glitches. For example, there are less glitches $(\approx 0 \sim 1)$ when we choose a challenge corresponding to a black element. The response is unstable when we choose a challenge corresponding to a white element. Note that the element 


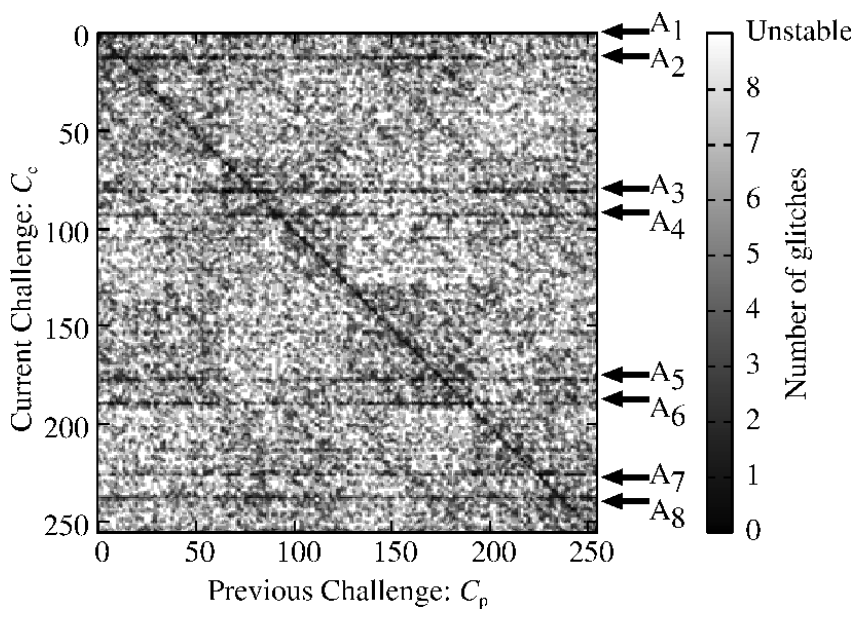

Fig. 12. Number of glitches (S-Box[6], Chip $i=1$ ).

means not the parity but the number of glitches. Naturally, a black diagonal line can be observed in this figure because no glitch occurs when both $C_{\mathrm{p}}$ and $C_{\mathrm{C}}$ are equal. Note that there are also a few black "horizontal lines", marked by arrows $\left(\mathrm{A}_{1} \sim \mathrm{A}_{8}\right)$. All 20 chips present the same pattern of lines. This means that some values of $C_{\mathrm{C}}$ lead to a small number of glitches independently of $C_{\mathrm{p}}$. Hence if we use such values of $C_{\mathrm{C}}$ as challenges to the GPUF, then adversaries will have the advantage of knowing that the number of glitches is small, which may help them succeed more easily with an attack aiming at predicting GPUF responses.

The following discusses the reason why such non-secure challenges exist using Fig. 13. An AES S-Box implementation using composite field consists of three sub-parts: isomorph $\delta$, Galois Field (GF) inverter, and a combination module of inverse isomorph $\delta^{-1}$ and affine transformation. Let the 8-bit variables $x$ and $y$ be the input and output of the AES S-Box, respectively. Also, let the 8-bit variables $a$ and $b$ be the outputs of the isomorph $\delta$ and the GF inverter, respectively. Our goal is to find special values of $x$ making the 6 -th output bit of the S-Box $(y[6])$ zero. In step 1 from Fig. 13, according to the properties of the combination module, the output value $y[6]$ satisfies:

$$
y[6]=\sim b[4] \oplus b[5] \oplus b[6] \oplus b[7] .
$$

Hence $y[6]$ depends on the upper 4 bits of $b$.

Next, in step 2 , we focus on the GF inverter. The value $b[7: 4]$, which represents the four most significant bits of $b$, satisfies:

$$
\begin{aligned}
& b[7]=\operatorname{tn}[0] \oplus \operatorname{tn}[1] \oplus \operatorname{tn}[3] \oplus \operatorname{tn}[4], \\
& b[6]=\operatorname{tn}[0] \oplus \operatorname{tn}[2] \oplus \operatorname{tn}[3] \oplus \operatorname{tn}[5], \\
& b[5]=\operatorname{tn}[0] \oplus \operatorname{tn}[1] \oplus \operatorname{tn}[7] \oplus \operatorname{tn}[8], \\
& b[4]=\operatorname{tn}[0] \oplus \operatorname{tn}[2] \oplus \operatorname{tn}[6] \oplus \operatorname{tn}[7] .
\end{aligned}
$$




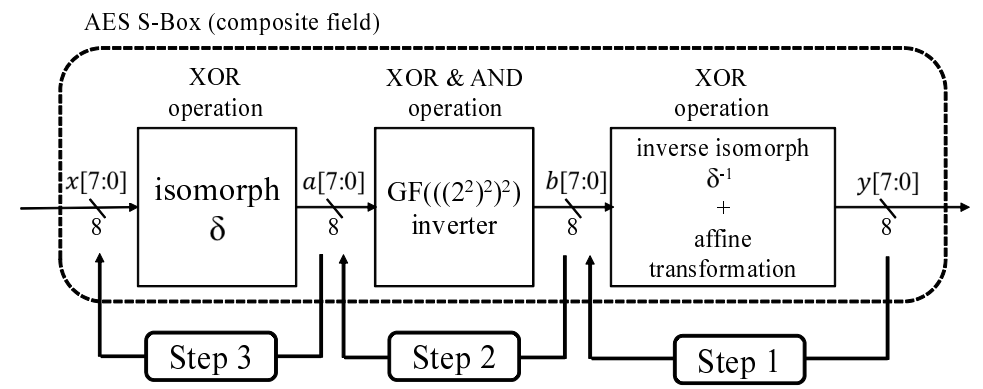

Fig. 13. An AES S-Box implementation using composite field.

Here, the 9-bit variable $t n$ is an internal variable in the GF inverter. The variable $t n$ satisfies:

$$
\begin{aligned}
& \operatorname{tn}[8]=\quad(v[3]) \quad \& \quad(a[7]), \\
& \operatorname{tn}[7]=\quad(v[2] \oplus v[3]) \quad \& \quad(a[6] \oplus a[7]), \\
& \operatorname{tn}[6]=\quad(v[2]) \quad \& \quad(a[6]), \\
& \operatorname{tn}[5]=\quad(v[1] \oplus v[3]) \quad \& \quad(a[5] \oplus a[7]), \\
& \operatorname{tn}[4]=(v[0] \oplus v[1] \oplus v[2] \oplus v[3]) \& \quad(a[4] \oplus a[5] \oplus a[6] \oplus a[7]), \\
& \operatorname{tn}[3]=\quad(v[0] \oplus v[2]) \quad \& \quad(a[4] \oplus a[6]), \\
& \operatorname{tn}[2]=\quad(v[1]) \quad \& \quad(a[5]), \\
& \operatorname{tn}[1]=\quad(v[0] \oplus v[1]) \quad \& \quad(a[4] \oplus a[5]), \\
& \operatorname{tn}[0]=\quad(v[0]) \quad \& \quad(a[4]) .
\end{aligned}
$$

The 4-bit variable $v$ is an internal variable in the GF inverter. Let us focus on the 4-bit variables, $a[4], a[5], a[6]$ and $a[7]$, on the right-hand side of the abovementioned equations of $t n$. If the values of the 4-bit variables are all zero, $t n$ also becomes zero. So the glitches caused by the variable $v$ do not propagate to $t n$, $b[7: 4]$ and $y[6]$. Consequently, if the most significant 4 bits of $a$ are zero, then no glitch is expected to appear in $y[6]$.

In step 3 , our goal is to find special values of $x$ which make $a[7: 4]$ equal to zero. The variable $a[7: 4]$ satisfies:

$$
\begin{aligned}
& a[7]=x[5] \oplus x[7], \\
& a[6]=x[1] \oplus x[2] \oplus x[3] \oplus x[4] \oplus x[6] \oplus x[7], \\
& a[5]=x[2] \oplus x[3] \oplus x[5] \oplus x[7], \\
& a[4]=x[1] \oplus x[2] \oplus x[3] \oplus x[5] \oplus x[7] .
\end{aligned}
$$


Hence the following holds:

$$
\begin{aligned}
x[1] & =0, \\
(x[5], x[7]) & =(0,0) \text { or }(1,1), \\
(x[2], x[3]) & =(0,0) \text { or }(1,1), \\
(x[4], x[6]) & = \begin{cases}(0,0) & \text { or }(1,1) \text { (if } x[7]=0), \\
(0,1) \text { or }(1,0) & \text { (if } x[7]=1) .\end{cases}
\end{aligned}
$$

Finally, we obtain the 16 patterns of the input $x$ that are expected to generate almost no glitches in the S-Box bit $y[6]$, as shown in Table 1. They correspond to the 16 specific values of $C_{\mathrm{C}}$ marked by the eight arrows in Fig. 12. There are actually 16 black horizontal lines in Fig. 12, but only eight lines corresponding to the eight arrows can be visually observed. This is because the 16 specific values consist of eight pairs of consecutive numbers. In our GPUF implementations, the number of glitches whose challenges $C_{\mathrm{c}}$ and $C_{\mathrm{p}}$ are one of the $16 \times 255$ patterns is zero or one, which is smaller than for other challenges. However, the GPUF responses include zero and one with almost the same ratio. This means that such 16 patterns of $C_{\mathrm{C}}$ are secure if the parity of the number of glitches is used as response. Some GPUFs implemented on other kinds of FPGAs or ASICs, however, have a possibility to generate no glitch if using the above values of $C_{\mathrm{C}}$. Hence we suggest that such values of $C_{\mathrm{C}}$ should not be used.

Table 1. The 16 patterns of the input $x$ generating almost no glitches in $y[6]$.

\begin{tabular}{c|c|c|c||c|l|c}
\hline$(x[5], x[7])$ & $(x[2], x[3])$ & $(x[4], x[6])$ & $x[1]$ & $x$ (binary) $(\dagger)$ & $x$ (decimal) & Arrows in Fig. 12 \\
\hline \hline \multirow{5}{*}{$(0,0)$} & \multirow{2}{*}{$(0,0)$} & $(0,0)$ & 0 & $0000000 *$ & 0,1 & $\mathrm{~A}_{1}$ \\
\cline { 3 - 7 } & \multirow{2}{*}{$(1,1)$} & $(1,1)$ & 0 & $0101000 *$ & 80,81 & $\mathrm{~A}_{3}$ \\
\cline { 3 - 7 } & & $(1,0)$ & 0 & $0000110 *$ & 12,13 & $\mathrm{~A}_{2}$ \\
\hline \multirow{3}{*}{$(1,1)$} & \multirow{2}{*}{$(0,0)$} & $(0,1)$ & 0 & $1110000 *$ & 224,225 & $\mathrm{~A}_{4}$ \\
\cline { 3 - 7 } & \multirow{2}{*}{$(1,1)$} & $(1,0)$ & 0 & $1011000 *$ & 176,177 & $\mathrm{~A}_{7}$ \\
\cline { 3 - 7 } & & $(1,0)$ & 0 & $1110110 *$ & 236,237 & $\mathrm{~A}_{8}$ \\
\hline
\end{tabular}

$(\dagger)$ Asterisks mean ' 0 ' or ' 1 '.

\section{Conclusion}

This paper experimentally analyzed GPUFs using a composite field-based AES S-Box implementation as a glitch generator on FPGAs. First, we clarified that the number of glitches depends on both the previous and current states of the registers dedicated to storing the challenge bits that are input to the AES SBox. As a consequence, GPUFs have a total of $2^{19}$ CRPs, which is much more 
than the $2^{11}$ CRPs evaluated by the GPUF developers [1] [16]. According to our experiments with 20 FPGAs, GPUFs using all $2^{19}$ CRPs showed a low robustness against voltage variation. Within the rated voltage range of the FPGAs $(1.14 \sim 1.26 \mathrm{~V})$, response error rates approached $35 \%$. The result exceeds the error correction range of a Fuzzy Extractor with a reasonable size of redundant data. Our results also indicated that GPUFs present almost no PUF-behavior as both reliability and uniqueness are relatively low. Finally, we found that our GPUF implementations have $16 \times 255$ weak challenges leading to almost no glitches regardless of the previous challenge bits stored in the registers. In conclusion, the AES S-Box implementation using composite field may not represent the best option for generating glitches for the GPUF due to issues with robustness against voltage variation and easily predictable responses.

To the best of our knowledge, other well-known AES S-Box implementations, such as sum of product (SOP), product of sum (POS), table lookup (TBL), positive polarity Reed-Miller (PPRM) [24] and 3-stage PPRM [25], are not suitable for GPUFs either. Although SOP, POS or TBL are able to generate glitches, these implementations have larger area size than a composite field-based implementation. Hence these designs are not suitable for PUFs on IC cards with limited resources. PPRM or 3-stage PPRM are designed to reduce the power consumption by preventing the generation of glitches. These are obviously not suitable for GPUFs. Thus we suggest that the AES S-Box should not be used as a glitch generator for GPUFs on FPGAs.

An ASIC implementation of the AES S-Box would probably not behave likewise FPGAs. The performance, such as reliability and uniqueness, has a possibility to improve if GPUFs are implemented on ASICs. Future work should include a discussion of performance and security evaluation of GPUFs on ASICs.

\section{Acknowledgements}

This work was supported in part by the Research Council KU Leuven: GOA TENSE (GOA/11/007), by the IAP Programme P6/26 BCRYPT of the Belgian State (Belgian Science Policy), by the European Commission through the ICT programme under contract ICT-2007-216676 ECRYPT II, by the Flemish Government through FWO G.0550.12N and the Hercules Foundation AKUL/11/19 and by the European Commission through the ICT programme under contract FP7-ICT-2011-284833 PUFFIN.

\section{References}

1. Daisuke Suzuki and Koichi Shimizu. The glitch puf: a new delay-puf architecture exploiting glitch shapes. In Proceedings of the 12th international conference on Cryptographic hardware and embedded systems, CHES'10, pages 366-382, Berlin, Heidelberg, 2010. Springer-Verlag.

2. Ravikanth S. Pappu. Physical one-way functions. PhD thesis, Massachusetts Institute of Technology, March 2001. 
3. B.Gassend, D.Clarke, M. van Dijk, and S.Devadas. Silicon physical random functions. In Proceedings of CCS '02, pages 148-160, 2002.

4. B.Gassend, D.Clarke, D. Lim, M. van Dijk, and S.Devadas. Identification and authentication of integrated circuits. In Concurrency and Computation: Practice and Experiences., pages 1077-1098, 2004.

5. R.Maes and I.Verbauwhede. Physically unclonable functions: A study on the state of the art and future research directions. In Towards Hardware Intrinsic Security: Foundation and Practice, Information Security and Cryptography, pages 3-37. Springer Berlin Heidelberg, 2010.

6. J.Guajardo, S. S. Kumar, G.J.Schrijen, and P.Tuyls. Fpga intrinsic pufs and their use for ip protection. In CHES 2007, pages 63-80, 2007.

7. D. E. Holcomb, W. P. Burleson, and K. Fu. Initial SRAM state as a fingerprint and source of true random numbers for RFID tags. In Proceedings of the Conference on RFID Security, July 2007.

8. S. S. Kumar, J.Guajardo, R.Maes, G.J.Schrijen, and P.Tuyls. The butterfly puf: Protecting ip on every fpga. In HOST, pages 67-70, 2008.

9. Roel Maes, Pim Tuyls, and Ingrid Verbauwhede. Intrinsic pufs from flip-flops on reconfigurable devices. In 3rd Benelux Workshop on Information and System Security (WISSec 2008), page 17, Eindhoven,NL, 2008.

10. Aswin Raghav Krishna, Seetharam Narasimhan, Xinmu Wang, and Swarup Bhunia. Mecca: A robust low-overhead puf using embedded memory array. In CHES, pages 407-420, 2011.

11. Y. Su, J. Holleman, and B. Otis. A 1.6pj/bit 96variations. In Solid-State Circuits Conference, 200\%. ISSCC 200\%. Digest of Technical Papers. IEEE International, pages 406-611, Feb. 2007.

12. Ying Su, J. Holleman, and B.P. Otis. A digital $1.6 \mathrm{pj} /$ bit chip identification circuit using process variations. Solid-State Circuits, IEEE Journal of, 43(1):69 -77, jan. 2008.

13. G. E. Suh and S. Devadas. Physical unclonable functions for device authentication and secret key generation. In Proceedings of DAC '07, pages 9-14, 2007.

14. Jae W. Lee, D. Lim, B. Gassend, G. E. Suh, M. Van Dijk, and S.Devadas. A technique to build a secret key in integrated circuits with identification and authentication applications. In Proceedings of the IEEE VLSI Circuits Symposium, pages $176-179,2004$.

15. Ulrich Rührmair, Frank Sehnke, Jan Sölter, Gideon Dror, Srinivas Devadas, and Jürgen Schmidhuber. Modeling attacks on physical unclonable functions. In Proceedings of the 17th ACM conference on Computer and communications security, CCS '10, pages 237-249, New York, NY, USA, 2010. ACM.

16. Koichi Shimizu, Daisuke Suzuki, and Tomomi Kasuya. Glitch puf: Extracting information from usually unwanted glitches. IEICE Transactions, 95-A(1):223$233,2012$.

17. James W. Crouch, Hiren J. Patel, Yong C. Kim, and Robert W. Bennington. Creating unique identifiers on field programmable gate arrays using natural processing variations. In $F P L$, pages 579-582, 2008.

18. Hiren J. Patel, James W. Crouch, Yong C. Kim, and Tony C. Kim. Creating a unique digital fingerprint using existing combinational logic. In $I S C A S$, pages 2693-2696, 2009.

19. Jason H. Anderson. A puf design for secure fpga-based embedded systems. In Proceedings of the 2010 Asia and South Pacific Design Automation Conference, ASPDAC '10, pages 1-6, Piscataway, NJ, USA, 2010. IEEE Press. 
20. Spartan-3E starter kit board. http://www.xilinx.com/products/devkits/HWSPAR3E-SK-US-G.htm.

21. AIST (Agency of Industrial Science, Technology), and Tohoku University in Japan. HDL code used for an AES S-Box implementation,. http://www.aoki.ecei. tohoku.ac.jp/crypto/items/AES_Comp.v.

22. Abhranil Maiti, Vikash Gunreddy, and Patrick Schaumont. A systematic method to evaluate and compare the performance of physical unclonable functions. Cryptology ePrint Archive, Report 2011/657, 2011.

23. R.Maes, P.Tuyls, and I.Verbauwhede. Low-overhead implementation of a soft decision helper data algorithm for sram pufs. In CHES 2009, pages 332-347, 2009.

24. T. Sasao. And-exor expressions and their optimization. In Logic Synthesis and Optimization, Kluwer Academic Publishers, pages 287-312, 1993.

25. Sumio Morioka and Akashi Satoh. An optimized s-box circuit architecture for low power aes design. In Cryptographic Hardware and Embedded Systems - CHES 2002, 4th International Workshop, Redwood Shores, CA, USA, August 13-15, 2002, Revised Papers, pages 172-186, 2002.

\section{Appendix}

In order to evaluate the reliability, we extract an $n$-bit $(65,280 \times 8)$ reference response $\left(R_{i}\right)$ from $i$-th FPGA chip $(1 \leq i \leq w, w=20$ in this work) at normal operating condition (room temperature and standard supply voltage of $1.20 \mathrm{~V}$ ). The same $n$-bit response is extracted at a different operating condition (different temperature and/or supply voltage) with a value $R_{i}^{\prime}$. Then, $m$ samples ( $m=100$ in this work, as in [1] [16]) of $R_{i}^{\prime}$ are collected. Here, $R_{i, t}^{\prime}$ is the $t$-th $(1 \leq t \leq m)$ sample of $R_{i}^{\prime}$. For the chip $i$ and the sample $t$, each data element of the reliability histogram is calculated as follows:

$$
\mathrm{HD}_{i, t}=\frac{1}{n} \sum_{C_{\mathrm{p}}=0}^{255} \sum_{C_{\mathrm{c}}=0}^{255} \operatorname{HD}\left\{R_{i}^{\prime}\left(C_{\mathrm{p}}, C_{\mathrm{c}}\right), R_{i, t}^{\prime}\left(C_{\mathrm{p}}, C_{\mathrm{c}}\right)\right\}
$$

Note that we exclude the responses where $C_{\mathrm{p}}$ equals $C_{\mathrm{c}}$ because no glitch occurs. The reliability histograms shown in Fig. 5 and Fig. 9 (I) include 2,000 data elements, resulted from $i$ and $t$. The response error rate shown in Fig. 8 and Fig. 10 (I) is calculated as follows:

$$
\text { ErrorRate }=\frac{1}{w \cdot m} \sum_{i=1}^{w} \sum_{t=1}^{m} \mathrm{HD}_{i, t} .
$$

